\title{
VIVÊNCIA DO PACIENTE ESTOMIZADO: UMA CONTRIBUIÇÃO PARA A ASSISTÊNCIA DE ENFERMAGEM
}

\author{
Conceição de Maria de Sá Nascimento ${ }^{1}$, Graciara Letícia Bezerra Trindade², Maria Helena Barros Araújo Luz \\ Roberta Fortes Santiago ${ }^{4}$
}

${ }^{1}$ Mestre em Enfermagem. Professora Adjunto do Colégio Agrícola da Universidade Federal do Piauí (UFPI). Piauí, Brasil. E-mail: consafran@hotmail.com

${ }^{2}$ Enfermeira. E-mail: graleticia@hotmail.com

${ }^{3}$ Doutora em Enfermagem. Professora Adjunto do Departamento de Enfermagem da UFPI. Piauí, Brasil. E-mail: mhelenal@ yahoo.com.br

${ }^{4}$ Mestre em Enfermagem. Enfermeira da Fundação Municipal de Saúde do Piauí. Piauí, Brasil. E-mail: betafortes@yahoo.com.br

\begin{abstract}
RESUMO: O presente trabalho foi desenvolvido com o objetivo de conhecer os significados atribuídos a vivência de pacientes estomizados, descrever seus conhecimentos sobre o autocuidado e identificar a importância das orientações de enfermagem para a sua adaptação. Pesquisa com abordagem qualitativa, realizada com 10 sujeitos associados ao programa de acompanhamento ao portador de estomia em um Centro de Referência a Estomizados, em Teresina-PI. Os dados foram obtidos por meio de entrevista e submetidos à análise de conteúdo, da qual emergiram três categorias que abordaram as mudanças no modo de vida do portador de estomia, $\mathrm{o}$ conhecimento do estomizado sobre o autocuidado e a orientação no processo de adaptação do portador de estomia. Concluiu-se que a estomia significa alterações no modo de vida e que a atuação da enfermagem através de atividades educativas é indispensável para o desenvolvimento do autocuidado e adaptação dos estomizados.
\end{abstract}

DESCRITORES: Estomia. Autocuidado. Adaptação psicológica. Enfermagem.

\section{THE OSTOMY PATIENT'S LIVING EXPERIENCE: A CONTRIBUTION TO NURSING CARE}

\begin{abstract}
This project was developed aiming to better understand the meanings attributed to ostomy patients' living experiences, to describe their knowledge of self-care, and to identify the importance of nursing guidelines in their adaptation. This research was qualitative in nature, performed with 10 subjects associated to the ostomy patient care program in a Reference Center for Ostomy Patients in Teresina, PI, Brazil. The data was collected through interviews and submitted to content analysis, from which three categories were obtained which outline the changes to the ostomized patient's way of life, their knowledge about self-care, and the orientation received in the adaptation process after an ostomy. We conclude that the ostomy procedure signifies lifestyle alterations and that nursing care through educational activities is indispensable to develop self-care and satisfactory ostomy adaptation.
\end{abstract}

DESCRIPTORS: Ostomy. Self care. Adaptation psychological. Nursing.

\section{LA EXPERIENCIA DEL PACIENTE OSTOMIZADO: UNA CONTRIBUCIÓN A LA ATENCIÓN DE ENFERMERÍA}

\begin{abstract}
RESUMEN: El presente estudio se hizo con el objetivo de conocer los significados que se atribuyen a la experiencia de los pacientes ostomizados, describir sus conocimientos sobre su propio cuidado y determinar la importancia de las orientaciones de la enfermería para su adaptación. Se trata de una investigación de carácter cualitativo, llevada a cabo con diez sujetos asociados al programa de atención a personas con ostomía en un Centro de Referencia para Pacientes Ostomizados, en la ciudad de Teresina-PI. Los datos recolectados por medio de entrevistas se examinaron según el análisis de contenido, dando lugar a tres categorías que abordan los cambios en el modo de vida de la persona con ostomía, el conocimiento del paciente ostomizado sobre su propio cuidado y la orientación sobre el proceso de adaptación de la persona con ostomía. Se concluyó que la ostomía significa un cambio en la forma de vida y que la enfermería por medio de actividades educativas es indispensable para el desarrollo del cuidado y la adaptación de los pacientes ostomizados.
\end{abstract}

DESCRIPTORES: Estomía. Autocuidado. Adaptación psicológica. Enfermería. 


\section{INTRODUÇÃO}

Os pacientes que sofrem agravo à saúde, no qual necessitam submeter-se a um procedimento cirúrgico para eliminar urina e/ou conteúdo fecal através da parede abdominal, rompendo com seu padrão habitual de eliminação, geralmente enfrentam dificuldades psicológicas e experimentam um sentimento repugnante em relação a si mesmo.

A palavra estoma deriva do grego, que significa uma abertura de qualquer víscera oca através do corpo, em situações diversas, recebendo denominações específicas, de acordo com o segmento a ser exteriorizado. As estomias podem ser temporárias ou definitivas. Quando essa exteriorização ocorre em algum segmento do intestino, para eliminação de fezes ou secreções, a denominamos de colostomia (abertura no cólon) e ileostomia (abertura no íleo). ${ }^{1}$

Pacientes submetidos a tal procedimento têm sua perspectiva de vida alterada, principalmente pela imagem corporal negativa, devido à presença do estoma associado a bolsa coletora. Além das mudanças nos padrões de eliminação, dos hábitos alimentares e de higiene precisam adaptar-se ao uso do equipamento, resultando em autoestima diminuída, sexualidade comprometida e, muitas vezes, em isolamento social.

Nesse contexto, cabe ao enfermeiro, como profissional de saúde, a compreensão dessas alterações, para desenvolver um plano de cuidados adequado ao preparo do paciente para o convívio com a estomia. O cuidar implica em uma interação entre o cuidador e quem está sendo cuidado, para troca de conhecimentos e experiências, proporcionando um resultado positivo de cuidado. ${ }^{2}$

Além dos problemas comumente enfrentados pelos pacientes que são submetidos a uma cirurgia, os estomizados enfrentam outros, tais como a exposição a uma série de constrangimentos sociais, pela possibilidade de saída dos gases e vazamento de excrementos mediante a inexistência de controle voluntário, e pela falha na segurança e qualidade da bolsa coletora, o que provoca o medo da exposição em público por parte desses pacientes. Normalmente tais problemas podem ser compreendidos sob as dimensões física, psicológica, social e espiritual. ${ }^{3}$

A atuação de enfermagem é de grande relevância, pois além de dar suporte no período que antecede à cirurgia, é uma ação de aprendizado em que o enfermeiro e o paciente interagem em uma relação empírica, buscando solucionar pro- blemas, por meio do diagnóstico de enfermagem. ${ }^{4}$ É também através da consulta de enfermagem que se tem um acompanhamento direto do paciente, prevenind o complicações relacionadas ao estoma, e ajudando-o a enfrentar as dificuldades ocasionadas pelas mudanças ocorridas após a estomização.

Para a enfermagem, a educação em saúde é indispensável e fundamental para o processo de cuidado, resultando em uma assistência de qualidade, pois o enfermeiro, além de cuidador é um educador, não apenas em relação aos demais membros da equipe de enfermagem, mas para o paciente, seus familiares e cuidador informal. ${ }^{5}$

A assistência ao paciente estomizado exige uma reflexão sobre os aspectos de reabilitação, significando um grande desafio para o profissional de saúde, principalmente de enfermagem, por isso é indispensável o conhecimento das necessidades desses pacientes através de suas indagações que, além de serem diversas, mudam constantemente. ${ }^{6}$

$\mathrm{O}$ interesse pela temática surgiu a partir do acompanhamento a portadores de estomias em um Centro de Referência em Teresina-PI, onde se observou que muitos não compareciam para a consulta de enfermagem, enviando terceiros para o recebimento das bolsas de estomia por receio de aparecerem em público. Assim, verificou-se a importância do comparecimento destes pacientes às consultas de enfermagem, a fim de conhecer suas vivências enquanto indivíduos estomizados e, desta forma, fornecer melhores orientações sobre a estomia e seus cuidados e contribuir para adaptação a situação vivenciada, de modo a proporcionar melhor qualidade de vida.

Este estudo é de grande relevância, pois segundo a Associação Brasileira de Ostomizados (ABRASO), ${ }^{7}$ estima-se que, no Brasil, há cerca de 50 mil estomizados, sendo que muitos desses são jovens, submetidos à cirurgia, após terem sido vítimas de traumatismos por arma de fogo, arma branca ou acidentes.

O processo de adaptação ocorre com o ajuste de toda uma vida, em um novo contexto, em que fatores importantes têm, muitas vezes, que serem abandonados, substituídos ou reduzidos. ${ }^{8}$ Portanto, é um processo individual que se desenvolve ao longo do tempo e envolve uma série de aspectos que vão desde a assistência oferecida, ao modo como o estomizado se envolve no próprio cuidado.

Acredita-se que a compreensão das pessoas estomizadas a partir de relato de suas vivências, possa proporcionar aos profissionais de enferma- 
gem uma perspectiva ampliada para orientar a elaboração de um cuidado apropriado.

De acordo com o exposto foi delimitado como objeto de pesquisa a vivência do paciente estomizado e teve como objetivos: conhecer os significados atribuídos à vivência dos portadores de estomias; descrever o conhecimento do estomizado sobre o autocuidado; e identificar a importância das orientações de enfermagem para o processo de adaptação destes pacientes.

\section{METODOLOGIA}

Trata-se de uma pesquisa exploratória e descritiva, com abordagem qualitativa, realizada com 10 pacientes estomizados, associados ao programa de acompanhamento ao portador de estomia em um Centro de Referência a Estomizados, em Teresina-PI.

Os critérios para seleção e inclusão dos sujeitos no estudo foram ser pacientes portadores de estomia temporária ou definitiva, com idade acima de dezoito anos e que concordassem em participar do estudo.

A coleta dos dados ocorreu em setembro de 2008 , mediante a utilização de um roteiro de entrevista semiestruturado, no qual os participantes responderam as seguintes perguntas: o que significa viver com uma estomia? Recebeu orientações dos profissionais? Quais eram e quem as prestavam? Como se sentiam ao realizar o autocuidado? Que dificuldades enfrentavam?.

Após a realização de cada entrevista, foi feita a escuta atenta da gravação e, posteriormente, a transcrição para a leitura textual. A finalização da coleta de dados deu-se quando ocorreu a saturação, levando-se em consideração a repetição dos conteúdos, falas, significados e singularidades das vivências.

A análise de dados ocorreu utilizando-se o método de análise de conteúdo, seguindo três etapas: pré-análise, exploração do material e tratamento dos resultados. ${ }^{9}$ Na pré-análise as entrevistas transcritas foram organizadas em quadros sistematizadores, seguida pela leitura flutuante, que permitiu ter uma visão geral dos dados coletados. A exploração do material aconteceu mediante análise detalhada do material organizado, o que permitiu sua codificação através de unidades temáticas relacionadas a vivência dos pacientes estomizados, o que possibilitou uma descrição das características pertinentes ao conteúdo por meio da identificação das unidades de significação. $\mathrm{Na}$ última etapa, a de tratamento e interpretação dos dados, as unidades de significação foram agrupadas em três categorias temáticas, as quais foram interpretadas a partir de estudos dos autores referenciados e de inferências que pudessem fundamentar melhor a discussão.

A pesquisa seguiu os critérios éticos, sendo aprovada pelo Comitê de Ética em Pesquisa da Faculdade Integral Diferencial (FACID), protocolo ${ }^{\circ}$ 442, respeitando a Resolução 196/96 do Conselho Nacional de Saúde, que regulamenta a pesquisa com seres humanos, permitindo aos participantes a livre escolha de aceitar ou não contribuir com o estudo, após o conhecimento de seus objetivos, metodologia, justificativa e condições de sua particpação assinando o Termo de Consentimento Livre e Esclarecido. A garantia da confidencialidade e anonimato também foi exposta e assegurada, uma vez que são utilizados pseudônimos com nomes de pedras preciosas.

\section{RESULTADOS E DISCUSSÃO}

Da análise das falas dos 10 participantes entrevistados, emergiram três categorias temáticas: mudanças no modo de vida do portador de estomia; o conhecimento do estomizado sobre o autocuidado e. a orientação no processo de adaptação do portador de estomia.

\section{Mudanças no modo de vida do portador de estomia}

Nesta categoria os entrevistados revelam que a estomia significa mudanças no seu modo de vida, em decorrência de dificuldades relacionadas ao trabalho, lazer, convívio social e familiar, sexualidade e alimentação, pela presença de sentimentos de vergonha e insegurança. As falas abaixo apontam dificuldades em relação ao trabalho:

[...] minha vida mudou porque eu não posso trabalhar nem fazer meus serviços dentro de casa [...] (Esmeralda).

[...] mudou na hora da gente sair para ir trabalhar, a gente se sente diferente [...] (Safira).

O impacto da estomia provoca uma alteração da imagem corporal, e ocorrem diversas reações, dependendo das características individuais, dos suportes sociais encontrados e da percepção da perda vivida pelo paciente. Estas pessoas enfrentam a perda da autoestima, o que pode levar a um sentimento de desprestígio diante da sociedade. ${ }^{10}$

O retorno às atividades ocupacionais/produtivas é dificultado pela sensação de perda ou redu- 
ção da capacidade produtiva. ${ }^{11}$ Tais dificuldades relacionadas ao trabalho e integração social, ocorrem devido ao uso da bolsa coletora causar desconforto, insegurança pela preocupação com os gases, vazamentos e eliminação de odor pelas fezes. ${ }^{8}$

Os participantes da pesquisa deixam clara a dificuldade de retorno às atividades de lazer, bem como às atividades de convívio social, principalmente no início, demonstrando superação com o passar do tempo:

[...] eu não saio porque de hora enquanto eu preciso lavar a bolsa, é dependioso (Esmeralda).

[...] ir ao dentista, ir ao salão, para sair a gente se sente incomodada, se acha diferente (Safira).

[...] eu não posso fazer uma viagem para outra cidade (Rubi).

[...] eu tinha vergonha de sair, para mim, eu iria me sujar [...] (Jade).

A maioria dos portadores de estomia não retoma ou retoma apenas parcialmente as atividades de lazer, como viajar ou praticar algum esporte, por insegurança, decorrente da qualidade dos dispositivos, ou ainda, por medo de problemas gastrintestinais. ${ }^{11}$

No viver do paciente estomizado existe um sentimento de impotência, representando uma decadência social, pois este sente-se incomodado apenas com um olhar, que lhes dá a sensação de estarem sendo julgados. ${ }^{12}$ Neste contexto, percebe-se que no âmbito social o estomizado tem a preocupação de manter secreta a estomia, até mesmo da própria família, como mostram as seguintes falas:

[...] Deus me livre, eu não quero jamais que as pessoas saibam, a maioria da família nem sabe [...] (Safira)

[...] minha família, acho que ninguém sabe, não quero causar preocupação (Esmeralda).

Observa-se que, muitas vezes, a pessoa estomizada incorpora um estigma social, ou seja, sente-se diferente perante sua família e sociedade, consequentemente dificultando sua própria aceitação e seu processo de adaptação, o que acarreta mudanças inclusive no relacionamento sexual, conforme relatam os entrevistados:

[...] mudou primeiramente o sexo, não tinha movimento, não é [...] (Opala).

[...] meu marido não me aceita [...] (Safira).

[...] sinto necessidade de fazer sexo, minha mulher mesmo não se sente bem, é só isso (Topázio).

Verifica-se nas falas que a sexualidade é outro aspecto afetado pela estomia, onde a maioria dos pacientes demonstrou ter diminuído sua atividade sexual devido a não aceitação ou recusa pelo parceiro.

As dificuldades sexuais enfrentadas são relacionadas às alterações na imagem corporal que tem início com a realização da cirurgia, a qual, pode causar algumas disfunções fisiológicas. No homem pode haver a redução ou mesmo a perda da libido, diminuição ou ausência da capacidade de ereção e alteração da ejaculação, e na mulher, redução ou perda da libido e, até mesmo, dispaurenia. ${ }^{6}$ As mesmas autoras acrescentam que boa parte das dificuldades sexuais também pode ter origem psicológica pela vergonha do parceiro, sensação de estar sujo e repugnante, o que gera medo de ser rejeitado.

De acordo com os entrevistados ocorrem também mudanças de hábitos essenciais como na alimentação e hidratação, o que representa também uma alteração no estilo de vida:

[...] não me alimento bem, porque tenho o problema do intestino não é, não posso comer uma comida forte, com caldo forte (Turquesa).

[...] o problema é da alimentação, para quem tem colostomia. No meu caso não posso beber muito para não urinar muito (Topázio).

As modificações nos hábitos alimentares têm o objetivo de evitar flatulência excessiva, além de complicações como diarréia, provocando abstinência de todos os alimentos que causam eliminação de gases. ${ }^{6}$ Ainda, apresentam o medo de alimentar-se em público, provocando a redução do prazer da alimentação e, consequentemente, o isolamento ou exclusão social.

Pessoas que passam pela estomização vivenciam modificações no seu dia-a-dia, não somente fisiológicas, como também de origem psicológica, emocional e social. ${ }^{13}$ Assim, fica claro que a compreensão das modificações ocorridas com o estomizado é fundamental, bem como o relato da vivência de todo esse processo, para oferecer um apoio efetivo.

\section{O conhecimento do estomizado sobre o autocuidado}

A partir dos relatos percebeu-se que os pacientes estomizados tinham certo conhecimento sobre o autocuidado em relação às técnicas de higienização, proteção da pele ao redor do estoma e da troca da bolsa de estomia. As falas a seguir evidenciam cuidados em relação à higiene.

O principal cuidado é limpar, não vestir roupa suja [...] (Turquesa). 
[...] lavar bem lavada, enxugar, ter bastante higiene (Safira).

O cuidado mais importante é a limpeza [...] (Ametista).

O mais importante é a parte da higiene não é, você tem que ter, para não pegar infecção (Diamante).

Cuidados com a troca de bolsa também foram citados e fazem parte do processo de reabilitação, uma vez que o indivíduo conhece e exerce as técnicas com o intuito de prevenir complicações e promover conforto e segurança. Nos depoimentos a seguir, fica evidente a importância desse cuidado na visão do estomizado.

Saber trocar a bolsa é um dos cuidados mais importantes [...] (Safira).

[...] trocar sempre a bolsa em dia, usar quatro a cinco dias no máximo [...] (Rubi).

Quando está suja, trocar a bolsa, se não estiver muito suja, só lavar [...] (Jade).

Ter o cuidado quando lavar a bolsa, secar direitinho. Para aderir, a pele é sensível demais, e se não tiver cuidado necessário, não consegue aderir (Ametista).

O cuidado com a troca da bolsa, de três em três dias no máximo, porque se passar de três dias fico muito sufocado, aquela urina esquenta e incomoda demais; se sente mal não é ? (Opala).

A literatura afirma que os cuidados com a higienização e a troca de bolsa das estomias são muito importantes para garantir a integridade da pele e prevenir infecção, e para que essas medidas sejam realizadas da maneira adequada, é preciso que os pacientes estomizados sejam orientados pelos profissionais de enfermagem, para que eles possam desenvolver o autocuidado. ${ }^{14}$

O paciente tem a oportunidade, através do autocuidado, de participar diretamente do seu processo de reabilitação e adaptação diante da nova situação. As atividades de autocuidado são definidas como habilidades humanas para engajamento em ações, de modo a promover a qualidade de vida, prevenir agravos e recuperar a saúde ou superar doenças e enfermidades. ${ }^{15}$

As aptidões da pessoa para o autocuidado, podem ter um papel decisivo na adaptação fisiológica, psicológica e social da pessoa estomizada e seus familiares ao processo de viver com uma estomia, o que contribui para a melhoria significativa da qualidade de vida destas pessoas. ${ }^{11}$

O conhecimento do autocuidado permite ao indivíduo maior independência em relação às outras pessoas, uma vez que ele próprio realiza seus cuidados através da prática de técnicas adequadas e simplificadas, que promovem a segurança do mesmo na realização do cuidado.

Ao se indagar sobre a segurança na realização do cuidado, fica evidente que as pessoas que têm o conhecimento sobre o autocuidado se sentem seguras para cuidar do estoma, como se pode ver nos depoimentos:

[...] me sinto segura, cuido bem. Graças a Deus, até agora não encontrei dificuldade, não é, graças a Deus (Rubi).

[...] me sinto seguro, faço tudo direitinho como o médico manda (Diamante).

[...] cuido muito bem, não tenho nenhum nervoso $e$ até hoje nunca tive problema nenhum com a estomia [...] (Turquesa).

Diversos fatores influenciam o autocuidado do paciente, bem como a adesão e motivação para o tratamento e as intervenções propostas. ${ }^{1}$ Vale ressaltar que a segurança no autocuidado foi demonstrado pelos depoentes que regularmente compareciam às consultas de enfermagem no Centro de Referência à Estomizados em Teresina-PI. Essas colocações levam-nos a considerar que a participação ativa do paciente junto à equipe multiprofissional é importantel para o desenvolvimento de suas habilidades e, portanto, contribuem para a sua adaptação, uma vez que através do autocuidado ocorre uma parceria, na qual os problemas são identificados e determinam as ações e o tipo de intervenção apropriada, tendo como resultado a segurança e reabilitação do paciente.

\section{A orientação no processo de adaptação do portador de estomia}

O paciente estomizado se depara com uma nova situação, uma vez que a imagem corporal construída durante toda a sua vida se quebra com a presença de um dispositivo na parede abdominal em que fezes ou urina são coletadas continuamente sem controle, significando a perda do controle esfincteriano e, consequentemente, gerando insegurança, medo, vergonha e isolamento social, necessitando assim de suporte para adaptação.

Ao analisar os depoimentos que correspondiam à adaptação, observou-se que alguns estomizados conviviam normalmente com o estoma.

Eu sou muito satisfeita com minha estomia, agradeço muito a Deus [...] eu fiquei outra pessoa. Antes eu só vivia escondida, vazava muita urina, mas com a estomia eu fiquei outra pessoa (Turquesa). 
Para mim eu levo uma vida normal, não há nada que eu não faça absolutamente (Topázio). tista).

Eu faço tudo na minha casa, ando normal (Ame-

Eu levo uma vida normal, como uma pessoa normal (Diamante).

No começo eu tive assim um preconceito, mas agora para mim eu nem ando com o estoma (Jade).

A adaptação tem a capacidade de modificar a relação entre o estresse e a enfermidade, resultando em controle dos fatores que levaram à inadaptabilidade. ${ }^{16} \mathrm{O}$ processo de adaptação leva à aceitação e convivência harmônica com a nova situação, como foi o caso dos depoimentos citados anteriormente. Porém, houve relatos de revolta e negação, relacionados à estomização, como se segue: (Safira).

[...] eu não aceito [...] me sinto revoltada mesmo

[...] se eu tivesse um jeito de mudar, eu iria mudar, tirar o estoma, mas não tenho condição [...] se eu tivesse a condição iria mandar voltar ao normal (Esmeralda).

[...] eu fiquei muito abatida não é, fico sem paciência [...] fico assim pensando que eu nunca tinha visto nem assim nos outros, tive a sorte de cair nessa (Rubi).

No processo de adaptação, o estomizado necessita de suportes que são definidos como as transformações interpessoais, que implicam na combinação de afeição, integração social, troca de mutualidade, senso de aliança segura e o significado de obtenção de orientação. ${ }^{17}$

Desse modo, as orientações necessárias ultrapassam a dimensão da transmissão de conhecimentos de modo verticalizado mas, uma busca de aproximação às suas inquietações, dúvidas e necessidade de lidar com as situações manifestadas.

Ao serem questionados a respeito de terem recebido orientações relacionadas à estomia, os depoentes se manifestaram do seguinte modo:

[...] recebi orientações [...] (Diamante).

[...] fui orientada e depois eu é quem já estava dando as orientações para as outras. eu dei muita orientação aqui para meus amigos e saiam todos satisfeitos [...] (Turquesa).

[...] fui orientada, o médico mandou que eu viesse procurar o programa e me orientaram [...] (Ametista).

Recebi orientação sim, nessa época era uma enfermeira e me explicou tudo direitinho (Jade).

A consulta de enfermagem é essencial por oferecer, além de suporte, a promoção de ações de aprendizagem, uma vez que permite uma relação empírica entre enfermeiro/paciente, buscando, através do diagnóstico de enfermagem, a solução de problemas. ${ }^{4}$ A orientação de enfermagem oferece um sentido, pois guia o paciente, neste caso o estomizado, para a aceitação pela compreensão das alterações ocorridas. Entretanto, nos depoimentos seguintes percebe-se que muitos estomizados não são orientados.

Não recebi nenhuma orientação (Esmeralda).

Não orientaram nada não [...] acho que devido ter tido alguma palestra e eu não fui assistir (Safira).

Não recebi orientação, só falaram que iriam tentar salvar minha vida (Ágata).

Orientação, não recebi (Opala).

Ao fazer uma análise dos depoimentos percebeu-se que os pacientes que receberam orientação foram os que compareciam frequentemente às consultas de enfermagem e exatamente aqueles que tiveram melhor aceitação e, consequentemente, adaptação à estomização, enquanto as pessoas que não foram orientadas tinham sentimento de negação e referiam alterações no estilo de vida. A literatura corrobora com a afirmação anterior, ao mencionar que a falta de uma assistência adequada, especialmente direcionada e articulada nas fases pré, trans e pós-operatório, envolvendo uma equipe multiprofissional, o próprio cliente e familiares, pode ocasionar dificuldade na aceitação da estomia, a adaptação dos novos hábitos de vida, além da rejeição da prática do autocuidado. ${ }^{18}$

Torna-se necessário que os enfermeiros desenvolvam atividades educativas em todos os encontros com pacientes estomizados e que criem maiores incentivos para o comparecimento destes às consultas, tais como dinâmicas interativas entre os pacientes que favoreçam a troca de vivências e experiências.

Os cuidados de enfermagem ao paciente estomizado devem ser iniciados no momento do diagnóstico e quando há indicação da realização da cirurgia, na expectativa de minimizar o sofrimento e obter melhor adaptação. ${ }^{19}$

As orientações devem englobar desde a realização do procedimento cirúrgico de estomização, bem como informações acerca de hábitos alimentares, higiene, possíveis complicações e, principalmente, sobre a importância do autocuidado que promove a independência do paciente, desenvolve o processo de adaptação ao estoma, além de prevenir complicações. No que diz respeito ao tipo de orientações pode-se perceber, 
principalmente, referentes ao autocuidado, como revelam as falas.

Tinha enfermeira, psicóloga e médico que orientavam a gente, sobre o uso da bolsa para manusear. Alguma coisa que eu fosse sentir, para procurá-los (Ametista).

Me ensinou como era que eu usava, o jeito que eu agia, quando sujasse, se eu pudesse lavar, eu lavava, se não pudesse, eu podia trocar (Jade).

[...] orientaram sempre que for tomar banho, lavar bem lavadinho, enxugar não é, quando estiver bem enxutinho, colocar (Opala).

A equipe multidisciplinar deve abordar o paciente e sua família com linguagem clara e de fácil entendimento, oferecendo orientações individualizadas, na busca de atender às expectativas dos mesmos. ${ }^{10}$

Dentre as orientações prestadas pelos membros da equipe multidisciplinar, a enfermagem tem papel preponderante, visto que está mais diretamente ligada a esta clientela. Portanto, sua participação é fundamental no processo de adaptação do estomizado, pois se o paciente receber orientação adequada e permanente, certamente irá sentir que está sendo bem cuidado e observado, logo, acatará melhor o tratamento, se mostrará mais seguro e interessado pela preservação do seu corpo e será mais colaborativo para o autocuidado.

\section{CONSIDERAÇÕES FINAIS}

A partir das vivências dos pacientes estomizados, esse estudo permitiu concluir que suas necessidades estavam geralmente relacionadas às mudanças ocorridas no modo de vida, pela não aceitação do estoma e pelo estigma causado por ele e que o enfermeiro como educador tem um papel fundamental para ajudar no enfrentamento desses problemas, como foi discutido nas categorias desta pesquisa.

As mudanças mais comuns estão relacionadas à dificuldade do retorno ao trabalho e às atividades de lazer. A sexualidade também foi descrita, por sentirem insegurança e medo da rejeição por parte do parceiro, sendo todos esses fatores geradores de alterações psicológicas, emocionas e sociais, provocando nestes pacientes o afastamento do convívio com outras pessoas, sendo necessários apoio emocional e desenvolvimento de estratégias de enfrentamento.

A orientação da equipe multidisciplinar é primordial para o desenvolvimento do autocuidado, promovendo, desta forma, independência e adaptação do paciente estomizado. As falas escla- receram que os pacientes que tiveram orientação acerca do quadro, bem como sobre técnicas de autocuidado, tiveram maior aceitação do estoma, conseguindo até mesmo levar uma vida normal. Em contrapartida, os pacientes que não receberam as devidas informações e orientações, apresentaram sentimento de revolta, comprovando o que traz a literatura, acerca da importância da orientação de enfermagem, que é majoritária no processo de reabilitação desses pacientes, quando permite a participação do mesmo no processo de cuidar.

Torna-se necessário que os enfermeiros desenvolvam atividades educativas em todos os encontros com pacientes estomizados e que criem maiores incentivos para o comparecimento destes às consultas, tais como dinâmicas interativas entre os pacientes, que favoreçam a troca de vivências e experiências.

Espera-se que o estudo contribua para o conhecimento e a compreensão dos estudantes e profissionais de enfermagem sobre a promoção de estratégias que leve a uma maior frequência dos estomizados às consultas, visando o desenvolvimento de atividades educativas permanentes, individuais e em grupo, para facilitar a adaptação destes, por meio de uma assistência qualificada.

\section{REFERÊNCIAS}

1. Santos VLCG. Estomaterapia através dos tempos. In: Santos VLCG; Cesaretti IUR. Assistência em estomaterapia cuidado do ostomizado. São Paulo (SP): Atheneu; 2005

2. Matheus MQ, Leite SMC, Dázio EMR. Compartilhando o cuidado da pessoa ostomizada. In: Anais do $2^{\circ}$ Congresso Brasileiro de Extensão Universitária [CD-ROM]. Belo Horizonte (MG): CEUFE, 2004 Set 12-15, 2004 [acesso 2008 Fev 28] Disponível em: http://www.ufmg.br/congrext/ Saude/Saude57.pdf

3. Gemelli LMG, Zago MMF. A interpretação do cuidado com o ostomizado na visão do enfermeiro: um estudo de caso. Revista Latino-am Enferm. 2002 Jan; 10(1):34-40.

4. Medonça RS, Valadão M, Castro L, Camargo TC. A importância da consulta de enfermagem em pré-operatório de ostomias intestinais. Rev Bras Cancerologia. 2007 Jan-Mar; 53(4):163-7.

5. Reveles AG, Takahashi RT. Educação em saúde ao estomizado: um estudo bibliométrico. Rev Esc Enferm USP. 2007 Jun; 41(2):245-50.

6. Silva AL, Shimizu HE. O significado da mudança no modo de vida da pessoa com estomia intestinal definitiva. Rev Latino-Am Enferm. 2006 Ago; 14(4):483-90. 
7. Associação Brasileira de Ostomizados. Declaração dos Direitos dos Ostomizados. Rio de Janeiro (RJ): ABRASO, 2003 [acesso 2008 Set 05]. Disponível em: http// www.abraso.org.br/ declaração.htm.

8. Menezes APS, Quintana JFA. Percepção do indivíduo estomizado quanto à sua situação. Rev Bras Prom Saúde. 2008 Fev; 21(1):13-8.

9. Bardin L. Análise de conteúdo. Lisboa (PT): Edições 70; 2008.

10. Barnabe NC, Dell'Acqua MCQ. Estratégias de enfrentamento (coping) de pessoas estomizadas. Revista Latino-am Enferm. 2008 Ago; 16(4):712-9.

11. Cascais AFMV, Martini JG; Almeida PJS. O impacto da ostomia no processo de viver humano. Texto Contexto Enferm. 2007 Jan-Mar; 16(1):163-7

12. Lucia SC. Sexualidade do ostomizado. In: Santos VLCG; Cesaretti IUR. Assistência em estomaterapia cuidado do ostomizado. São Paulo (SP): Atheneu; 2005.

13. Sonobe HM, Barichello E, Zago MMA. Visão do colostomizado sobre o uso da bolsa de colostomia. Rev Bras Cancerologia. 2002 Jul-Set; 48(3):341-8.
14. Timy BK, Smith NE. Enfermagem médico-cirúrgica. São Paulo (SP): Editora Manole; 2005.

15. Ávila C, Petuco VM. O paciente ostomizado: visão da equipe de enfermagem. Rev Médica HSVP. 2001; 11(27):46-54.

16. Castro ME. Adaptação do ostomizado como processo de desenvolvimento humano: abordagem do modelo de Callista Roy. $1^{\text {a }}$ ed. Fortaleza (CE): Gráfica LCR, 2001.

17. Santos VLCG. Fundamentação teórico- metodológica da assistência aos ostomizados na área da saúde do adulto. RevEsc Enferm USP. 2000 Mar; 34(1):59-63.

18. Santos VLCG, Sawaia BB. A bolsa de na medição "estar ostomizado"-"estar profissional" análise de uma estratégia pedagógica. Rev Latino-am Enfermagem. 2000 Jul; 8(3):40-50.

19. Tosato SR; Zimmermann MH. Conhecimento do indivíduo ostomizado em relação ao autocuidado. Rev Conexão. 2005 [acesso 2009 Nov 17]; 2: 34-7. Disponível em: http://www.uepg.br/ revistaconexao/revista/edicao02/edicao02\%20-\%20 34-37\%20conhecimento\%20do\%20individuo.pdf 